\title{
BREAST SCHWANNOMA - A RARE ENTITY
}

Juliana Lopes de Aguiar Araujo1', Ubiratan Wagner de Sousa1', Ana Tereza Diniz Marinho de França², Lourdes Maria Dantas de Góis

${ }^{1}$ Liga Norte Rio-Grandense Contra O Câncer - Natal (RN), Brazil.

2Universidade Potiguar - Natal (RN), Brazil.

Introduction: Schwannoma is a benign tumor originating from Schwann cells present in the myelin sheath of peripheral nerves. Breast presentation is rare, and its clinical manifestation can mimic that of breast carcinoma. Objectives: To report the case of a patient with breast Schwannoma. Method/Case report: T.S.L., 14 years old, presented a nodule in the left breast (LB) with local growth for 2 months. Ultrasound (US) revealed a cystic formation in the lower outer quadrant of 1.8x1.3 cm, BI-RADS 2. Physical examination indicated a nodular skin lesion next to the LB fold, with $2 \mathrm{~cm}$ at $5 \mathrm{~h}, 7$ $\mathrm{cm}$ from the nipple. After the surgical excision of the nodule, anatomopathological examination showed a spindle cell neoplasm without nodular-pattern atypia or malignancy characteristics. Immunohistochemistry (IHC) confirmed the Schwannoma diagnosis. Considering this scenario, annual control was started, and the patient has no evidence of the disease after 5 years of diagnosis. Results/Discussion: Schwannoma is a typically benign tumor originating from Schwann cells in the myelin sheath of the nerves. It may result from the parasympathetic or sympathetic division of the autonomic nervous system of the organ. It is one of the few truly encapsulated tumors of the human body and almost always solitary. It is usually located in the trunk, flexor surfaces, retroperitoneum, and rarely in the breast, representing approximately $2.6 \%$ of Schwannoma cases. It affects individuals aged 30 to 50 years, with equal incidence in both men and women. The skin lesion presents as a sessile, asymptomatic nodule of $1-3 \mathrm{~cm}$ and slow growth. Pain and sensitivity may be present when tumor growth causes nerve compression. Mammography (MMG) shows well-circumscribed opacity, with a density similar to that of soft tissue. US usually reveals well-defined, hypoechoic, solid lesions and can include the target sign, posterior acoustic enhancement, and continuity with peripheral nerves. The definitive diagnosis is made histologically, and the differential diagnosis involves other spindle cell neoplasms, such as fibroadenoma, phyllodes tumor, leiomyoma, fibromatosis, and, rarely, metaplastic carcinoma. Fine-needle aspiration biopsy (FNAB) showed palisading fibrillar cells with non-atypical spindle-shaped nuclei forming Verocay bodies. IHC indicates intense and uniform expression of S-100 protein. Malignant transformation occurs in 3-10\% of cases, with high cellular proliferation, atypical mitotic activity, cellular and nuclear pleomorphism, and foci of necrosis. The treatment of choice is surgical excision of the lesion. Tumor recurrence is low and associated with the mitotic index. Conclusion: The case reported and publications found bring to light the discussion on diagnosis and treatment of breast Schwannoma, a rare and benign neoplasm in this location. 